\title{
A note on cone $b$-metric and its related results: generalizations or equivalence?
}

Wei-Shih Du ${ }^{1}$ and Erdal Karapınar ${ }^{2 *}$

"Correspondence: erdalkarapinar@yahoo.com; ekarapinar@atilim.edu.tr ${ }^{2}$ Department of Mathematics, Atilim University, İncek, Ankara 06836, Turkey

Full list of author information is available at the end of the article

\begin{abstract}
Very recently, a notion of cone $b$-metric was introduced as a generalization of $b$-metric, and some related fixed point results were obtained. In this paper, we investigate the answer to the question whether the given results generalize the existing ones or are equivalent to them.
\end{abstract}

MSC: $46 \mathrm{~N} 40 ; 47 \mathrm{H} 10 ; 54 \mathrm{H} 25 ; 46 \mathrm{~T} 99$

Keywords: TVS-cone metric space; $b$-metric; TVS-cone $b$-metric; nonlinear scalarization function; fixed point

\section{Introduction and preliminaries}

Topological vector space-valued metric space (or TVS-cone metric space) introduced by $\mathrm{Du}$ [1] as a generalization of the Banach-valued/cone metric space [2]. Recently, Du [1] noted that fixed point theorems in generalized cone metric spaces and in usual metric spaces are equivalent. In particular, the author proved that the celebrated fixed point theorems of Banach, Kannan, Chatterjea, etc. in both TVS-cone metric can be derived easily from the usual metric space set-up, by a simple manipulation, namely, using a scalarization function. Very recently, a number of publications, dealing with the cone $b$-metric space structure and fixed point theorems on such spaces, appeared. In this paper, we show that fixed point theorems in cone $b$-metric and usual $b$-metric spaces are equivalent. This paper can be considered as a continuation of the report [1].

A topological vector space (t.v.s. for short) is a vector space with a topology such that the vector space operations (addition and scalar multiplication) are continuous. A topological vector space is locally convex if its origin has a basis of neighborhoods that are convex. Let $Y$ be a locally convex Hausdorff t.v.s. with its zero vector $\theta$, let $\tau$ denote the topology of $Y$, and let $\mathcal{U}_{\tau}$ be the base at $\theta$, consisting of all absolutely convex neighborhood of $\theta$. Let

$$
\mathcal{L}=\left\{\ell: \ell \text { is a Minkowski functional of } U \text { for } U \in \mathcal{U}_{\tau}\right\}
$$

Then $\mathcal{L}$ is a family of seminorms on $Y$. For each $\ell \in \mathcal{L}$, let

$$
V(\ell)=\{y \in Y: \ell(y)<1\}
$$

and let

$$
\begin{aligned}
\mathcal{U}_{\mathcal{L}}= & \left\{U: U=r_{1} V\left(\ell_{1}\right) \cap r_{2} V\left(\ell_{2}\right) \cap \cdots \cap r_{n} V\left(\ell_{n}\right),\right. \\
& \left.r_{k}>0, \ell_{k} \in \mathcal{L}, 1 \leq k \leq n, n \in \mathbb{N}\right\}
\end{aligned}
$$

(0) 2013 Du and Karapınar; licensee Springer. This is an Open Access article distributed under the terms of the Creative Commons Attribution License (http://creativecommons.org/licenses/by/2.0), which permits unrestricted use, distribution, and reproduction in any medium, provided the original work is properly cited. 
Then $\mathcal{U}_{\mathcal{L}}$ is a base at $\theta$, and the topology $\Gamma_{\mathcal{L}}$ generated by $\mathcal{U}_{\mathcal{L}}$ is the weakest topology for $Y$ such that all seminorms in $\mathcal{L}$ are continuous and $\tau=\Gamma_{\mathcal{L}}$. Moreover, given any neighborhood $\mathcal{O}_{\theta}$ of $\theta$, there exists $U \in \mathcal{U}_{\mathcal{L}}$ such that $\theta \in U \subset \mathcal{O}_{\theta}$ (see, e.g., [3, Theorem 12.4 in II.12, p.113]).

Throughout this paper, we follow all notations considered in [1]. Let $E$ be a t.v.s. with its zero vector $\theta_{E}$. A nonempty subset $K$ of $E$ is called a cone if $\lambda K \subseteq K$ for $\lambda \geq 0$. A cone $K$ is said to be pointed if $K \cap(-K)=\left\{\theta_{E}\right\}$. For a given cone $K \subseteq E$, we can define a partial ordering $\precsim$ (or $\precsim_{K}$ ) with respect to $K$ by

$$
x \precsim y \Longleftrightarrow y-x \in K .
$$

$x \prec y$ will stand for $x \precsim y$ and $x \neq y$, while $x \ll y$ will stand for $y-x \in \operatorname{int} K$, where int $K$ denotes the interior of $K$.

Let $E$ be a t.v.s. and $K$ a convex cone with int $K \neq \emptyset$ in $E$. Then it is obvious that

$$
\operatorname{int} K+\operatorname{int} K \subseteq \operatorname{int} K+K \subseteq \operatorname{int} K
$$

and

$\lambda \operatorname{int} K \subseteq \operatorname{int} K \quad$ for all $\lambda>0$.

In the following, unless otherwise specified, we always assume that $Y$ is a locally convex Hausdorff t.v.s. with its zero vector $\theta, K$ a proper, closed and convex pointed cone in $Y$ with int $K \neq \emptyset, e \in \operatorname{int} K$ and $\precsim$ a partial ordering with respect to $K$.

The nonlinear scalarization function $[1,4,5] \xi_{e}: Y \rightarrow \mathbb{R}$ is defined as follows:

$$
\xi_{e}(y)=\inf \{r \in \mathbb{R}: y \in r e-K\} \quad \text { for all } y \in Y .
$$

Lemma 1.1 (See, e.g., $[1,4,5])$ For each $r \in \mathbb{R}$ and $y \in Y$, the following statements are satisfied:

(i) $\xi_{e}(y) \leq r \Longleftrightarrow y \in r e-K$,

(ii) $\xi_{e}(y)>r \Longleftrightarrow y \notin r e-K$,

(iii) $\xi_{e}(y) \geq r \Longleftrightarrow y \notin r e-\operatorname{int} K$,

(iv) $\xi_{e}(y)<r \Longleftrightarrow y \in r e-\operatorname{int} K$,

(v) $\xi_{e}(\cdot)$ is positively homogeneous and continuous on $Y$,

(vi) if $y_{1} \in y_{2}+K$ (i.e. $\left.y_{2} \precsim y_{1}\right)$, then $\xi_{e}\left(y_{2}\right) \leq \xi_{e}\left(y_{1}\right)$,

(vii) $\xi_{e}\left(y_{1}+y_{2}\right) \leq \xi_{e}\left(y_{1}\right)+\xi_{e}\left(y_{2}\right)$ for all $y_{1}, y_{2} \in Y$.

\section{Remark 1.2}

(a) Clearly, $\xi_{e}(\theta)=0$.

(b) It is worth mentioning that the reverse statement of (vi) in Lemma 1.1 (i.e., $\left.\xi_{e}\left(y_{2}\right) \leq \xi_{e}\left(y_{1}\right) \Longrightarrow y_{2} \precsim y_{1}\right)$ does not hold in general. For example, let $Y=\mathbb{R}^{2}$, $K=\mathbb{R}_{+}^{2}=\left\{(x, y) \in \mathbb{R}^{2}: x, y \geq 0\right\}$, and let $e=(1,1)$. Then $K$ is a proper, closed, convex and pointed cone in $Y$ with int $K=\left\{(x, y) \in \mathbb{R}^{2}: x, y>0\right\} \neq \emptyset$ and $e \in \operatorname{int} K$. For $r=1$, it is easy to see that $y_{1}=(8,-15) \notin r e-\operatorname{int} K$, and $y_{2}=(0,0) \in r e-\operatorname{int} K$. By applying (iii) and (iv) of Lemma 1.1, we have $\xi_{e}\left(y_{2}\right)<1 \leq \xi_{e}\left(y_{1}\right)$, while $y_{1} \notin y_{2}+K$. 


\subsection{TVS-cone metric spaces}

Definition 1.3 (See [1]) Let $X$ be a nonempty set. Suppose that a vector-valued function $p: X \times X \rightarrow Y$ satisfies:

(C1) $\theta \precsim p(x, y)$ for all $x, y \in X$ and $p(x, y)=\theta$ if and only if $x=y$,

(C2) $p(x, y)=p(y, x)$ for all $x, y \in X$,

(C3) $p(x, y) \precsim p(x, z)+p(z, y)$ for all $x, y, z \in X$.

Then, the function $p$ is called a TVS-cone metric on $X$. Furthermore, the pair $(X, p)$ is called a TVS-cone metric space (in short, TVS-CMS).

Lemma 1.4 (See [1]) Let $(X, p)$ be a TVS-CMS. Then, $d_{p}: X \times X \rightarrow[0, \infty)$ defined by $d_{p}=\xi_{e} \circ p$ is a metric.

Remark 1.5 We notice that a cone metric space (in short, CMS), introduced by Huang and Zhang [2], is a special case of TVS-CMS. Indeed, the authors [2] considered $E$ as a real Banach space instead of TVS in Definition 1.3. Further, for a CMS $(X, p)$, the function $d_{p}: X \times X \rightarrow[0, \infty)$ defined by $d_{p}=\xi_{e} \circ p$ is also a metric.

Definition 1.6 (See [1]) Let $(X, p)$ be a TVS-CMS, $x \in X$ and $\left\{x_{n}\right\}_{n \in \mathbb{N}}$ a sequence in $X$.

(i) $\left\{x_{n}\right\}_{n \in \mathbb{N}}$ TVS-cone converges to $x \in X$ whenever for every $\theta \ll c \in Y$, there is a natural number $M$ such that $p\left(x_{n}, x\right) \ll c$ for all $n \geq M$ and denoted by cone- $\lim _{n \rightarrow \infty} x_{n}=x$ (or $x_{n} \stackrel{\text { cone }}{\longrightarrow} x$ as $\left.n \rightarrow \infty\right)$,

(ii) $\left\{x_{n}\right\}_{n \in \mathbb{N}}$ TVS-cone Cauchy sequence in $(X, p)$ whenever for every $\theta \ll c \in Y$, there is a natural number $M$ such that $p\left(x_{n}, x_{m}\right) \ll c$ for all $n, m \geq M$,

(iii) $(X, p)$ is TVS-cone complete if every sequence TVS-cone Cauchy sequence in $X$ is a TVS-cone convergent.

Theorem 1.7 (See $[1,6])$ Let $(X, p)$ be a TVS-CMS, $x \in X$, and let $\left\{x_{n}\right\}_{n \in \mathbb{N}}$ be a sequence in $X$. Set $d_{p}=\xi_{e} \circ p$. Then the following statements hold:

(i) $\left\{x_{n}\right\}_{n \in \mathbb{N}}$ converges to $x$ in TVS-CMS $(X, p)$ if and only if $d_{p}\left(x_{n}, x\right) \rightarrow 0$ as $n \rightarrow \infty$,

(ii) $\left\{x_{n}\right\}_{n \in \mathbb{N}}$ is a Cauchy sequence in TVS-CMS $(X, p)$ if and only if $\left\{x_{n}\right\}_{n \in \mathbb{N}}$ is a Cauchy sequence in $\left(X, d_{p}\right)$,

(iii) $(X, p)$ is a complete TVS-CMS if and only if $\left(X, d_{p}\right)$ is a complete metric space.

Remark 1.8 From Theorem 1.7, we conclude that for every complete TVS-cone metric space, there exists a correspondent isomorphic complete usual metric space. Notice that the cone should have a nonempty interior.

Proposition 1.9 (See [1]) Let $(X, p)$ be a complete TVS-CMS and $0 \leq \gamma<1$. If $T: X \rightarrow X$ satisfies the contractive condition

$$
p(T x, T y) \precsim \gamma p(x, y) \quad \text { for all } x, y \in X,
$$

then $T$ has a unique fixed point in $X$. Moreover, for each $x \in X$, the iterative sequence $\left\{T^{n} x\right\}_{n=1}^{\infty}$ converges to the unique fixed point of $T$.

In particular, if $K$ is a cone of a real Banach space $V$, then it is called normal if there is a number $\rho \geq 1$ such that for all $x, y \in V: \theta \precsim x \precsim y \Longrightarrow\|x\| \leq \rho\|y\|$. The least positive integer $\rho$, satisfying this inequality, is called the normal constant of $K$. 


\section{$1.2 b$-Metric spaces}

The notion of a $b$-metric space was considered by Bakhtin [7] and Czerwik [8] as a generalization of metric space.

Definition 1.10 (See [7-10]) Let $X$ be a nonempty set, and let $s \geq 1$ be a given real number. A function $d: X \times X \rightarrow[0, \infty)$ is called a $b$-metric if the following conditions are satisfied:

(1) $d(x, y)=0$ if and only if $x=y$;

(2) $d(x, y)=d(y, x)$

(3) $d(x, z) \leq s[d(x, y)+d(y, z)]$ for all $x, y, z \in X$.

A pair $(X, d)$ is called a $b$-metric space.

In this paper, we first introduce the concept of TVS-cone $b$-metric space which generalize the concept of $b$-metric space and cone $b$-metric space.

Definition 1.11 Let $X$ be a non-empty set and $s \geq 1$ be a given real number. A vectorvalued function $p: X \times X \rightarrow Y$ is said to be TVS-cone $b$-metric if the following conditions are satisfied:

(BM1) $\theta \precsim p(x, y)$ for all $x, y \in X$ and $p(x, y)=\theta$ if and only if $x=y$;

(BM2) $p(x, y)=p(y, x)$;

(BM3) $p(x, z) \precsim s[p(x, y)+p(y, z)]$ for all $x, y, z \in X$.

The pair $(X, p)$ is called a TVS-cone $b$-metric space.

If we replace $Y$ by a real Banach space in Definition 1.11, we get the cone $b$-metric space in the sense of [11-13]. It is evident that Definition 1.10 coincides with Definition 1.11 if we replace $Y$ by a set of non-negative real numbers.

\section{Main results}

The following theorem is one of main results in this paper. Although it is the mimic of the proof of Lemma 1.4, we give the proof for the sake of completeness and for the readers' convenience.

Theorem 2.1 Let $(X, p)$ be a TVS-cone b-metric space. Then, $d_{p}: X \times X \rightarrow[0, \infty)$ defined by $d_{p}=\xi_{e} \circ p$ is a b-metric.

Proof Clearly, $d_{p}(x, y)=d_{p}(y, x)$ for all $x, y \in X$. By Lemma 1.1, we have $d_{p}(x, y) \geq 0$ for all $x, y \in X$. If $x=y$, then, by (BM1), $d_{p}(x, y)=\xi_{e}(\theta)=0$. Conversely, if $d_{p}(x, y)=0$, then by Lemma 1.1 $p(x, y) \in K \cap(-K)=\{\theta\}$, which implies that $x=y$. Since $s \geq 1$, by applying (v), (vi) and (vii) of Lemma 1.1, we have

$$
\xi_{e}(p(x, z)) \leq s\left(\xi_{e}(p(x, y))+\xi_{e}(p(y, z))\right)
$$

or

$$
d_{p}(x, z) \leq s\left[d_{p}(x, y)+d_{p}(y, z)\right] \quad \text { for all } x, y, z \in X .
$$

So we prove that $d_{p}$ is a $b$-metric.

The following consequence of Theorem 2.1 is evident. 
Corollary 2.2 Let $(X, p)$ be a cone b-metric space. Then, $d_{p}: X \times X \rightarrow[0, \infty)$ defined by $d_{p}=\xi_{e} \circ p$ is a b-metric.

Following the idea of $\mathrm{Du}[1]$, we can define the following.

Definition 2.3 Let $(X, p)$ be a TVS-cone $b$-metric space, let $x \in X$, and let $\left\{x_{n}\right\}_{n \in \mathbb{N}}$ be a sequence in $X$.

(i) $\left\{x_{n}\right\}_{n \in \mathbb{N}}$ TVS-cone converges to $x \in X$ whenever for every $\theta \ll c \in Y$, there is a natural number $M$ such that $p\left(x_{n}, x\right) \ll c$ for all $n \geq M$ and denoted by cone- $\lim _{n \rightarrow \infty} x_{n}=x$ (or $x_{n} \stackrel{\text { cone }}{\longrightarrow} x$ as $\left.n \rightarrow \infty\right)$,

(ii) $\left\{x_{n}\right\}_{n \in \mathbb{N}}$ TVS-cone Cauchy sequence in $(X, p)$ whenever for every $\theta \ll c \in Y$, there is a natural number $M$ such that $p\left(x_{n}, x_{m}\right) \ll c$ for all $n, m \geq M$,

(iii) $(X, p)$ is TVS-cone complete if every sequence TVS-cone Cauchy sequence in $X$ is a TVS-cone convergent.

Using a similar argument as in the proof of [2, Theorem 2.2], we can prove the following result.

Theorem 2.4 Let $(X, p)$ be a TVS-cone b-metric space, let $x \in X$, let and $\left\{x_{n}\right\}_{n=1}^{\infty}$ be a sequence in $X$. Set $d_{p}=\xi_{e} \circ p$. Then the following statements hold:

(i) $\left\{x_{n}\right\}_{n \in \mathbb{N}}$ converges to $x$ in TVS-cone $b$-metric space $(X, p)$ if and only if $d_{p}\left(x_{n}, x\right) \rightarrow 0$ as $n \rightarrow \infty$,

(ii) $\left\{x_{n}\right\}_{n \in \mathbb{N}}$ is a Cauchy sequence in TVS-cone b-metric space $(X, p)$ if and only if $\left\{x_{n}\right\}_{n \in \mathbb{N}}$ is a Cauchy sequence in $\left(X, d_{p}\right)$,

(iii) $(X, p)$ is a complete TVS-cone $b$-metric space if and only if $\left(X, d_{p}\right)$ is a complete $b$-metric space.

Remark 2.5 From Theorem 2.4, we conclude that for every complete TVS-cone $b$-metric space there exists a correspondent isomorphic complete usual (associated) $b$-metric space.

Theorem 2.6 Let $(X, p)$ be a complete TVS-cone b-metric space with $s \geq 1$ and $0 \leq \gamma<1$. If $T: X \rightarrow X$ satisfies the contractive condition

$$
p(T x, T y) \precsim \gamma p(x, y) \quad \text { for all } x, y \in X,
$$

then $T$ has a unique fixed point in $X$. Moreover, for each $x \in X$, the iterative sequence $\left\{T^{n} x\right\}_{n \in \mathbb{N}}$ converges to the unique fixed point of $T$.

Proof Set $d_{p}=\xi_{e} \circ p$. Due to Theorem 2.4, we conclude that $\left(X, d_{p}\right)$ is a complete $b$-metric space. On the other hand, from Lemma 1.1, we derive that

$$
p(T x, T y) \precsim \gamma p(x, y) \quad \Longrightarrow \quad d_{p}(T x, T y) \leq \gamma d_{p}(x, y) \quad \text { for all } x, y \in X .
$$

We conclude the results from the characterization of the Banach contraction mapping principle in the context of $b$-metric space (see, e.g., [14, Theorem 2]). The proof is completed. 
Theorem 2.7 Let $(X, p)$ be a complete TVS-cone b-metric space with $s \geq 1$, and let $T$ : $X \rightarrow X$ satisfy the contractive condition

$$
p(T x, T y) \precsim \lambda_{1} p(x, T x)+\lambda_{2} p(y, T y)+\lambda_{3} p(x, T y)+\lambda_{4} p(y, T x) \quad \text { for all } x, y \in X,
$$

where $\lambda_{i} \in[0,1), i=1,2,3,4$, and $\lambda_{1}+\lambda_{2}+s\left(\lambda_{3}+\lambda_{4}\right)<\min \left\{1, \frac{2}{s}\right\}$. Then $T$ has a unique fixed point in $X$. Moreover, for each $x \in X$, the iterative sequence $\left\{T^{n} x\right\}_{n \in \mathbb{N}}$ converges to the unique fixed point of $T$.

The idea of the proof is the same with the proof of Theorem 2.6. For the sake of completeness, we put it here.

Proof Set $d_{p}=\xi_{e} \circ p$. Due to Theorem 2.4, we conclude that $\left(X, d_{p}\right)$ is a complete $b$-metric space. On the other hand, from Lemma 1.1, we derive that

$$
p(T x, T y) \precsim \lambda_{1} p(x, T x)+\lambda_{2} p(y, T y)+\lambda_{3} p(x, T y)+\lambda_{4} p(y, T x)
$$

implies that

$$
d_{p}(T x, T y) \precsim \lambda_{1} d_{p}(x, T x)+\lambda_{2} d_{p}(y, T y)+\lambda_{3} d_{p}(x, T y)+\lambda_{4} d(y, T x) \quad \text { for all } x, y \in X .
$$

We conclude the result from [14, Corollary 4.1] with taking $S=T$. The proof is completed.

\section{Conclusion}

In this paper, we just show that two fixed point theorems in the setting of cone $b$-metric spaces can be easily derived from the existing result in the context of $b$-metric space. Hence, the notion of 'cone $b$-metric' is not a real generalization of neither $b$-metric nor metric. By using the techniques above, one can easily prove the equivalence of other fixed point results (published, unpublished/that will be published) in the context of cone $b$-metric space. Regarding the published papers on the equivalence of cone metric and usual (associated) metric in the literature, it is natural to conclude that some other techniques can also be developed for the equivalence of the mentioned notions.

Competing interests

The authors declare that they have no competing interests.

Authors' contributions

Both authors contributed equally and significantly in writing this paper. Both authors read and approved the final manuscript.

\section{Author details}

${ }^{1}$ Department of Mathematics, National Kaohsiung Normal University, Kaohsiung, 824, Taiwan. ${ }^{2}$ Department of Mathematics, Atilim University, Incek, Ankara 06836, Turkey.

\section{Acknowledgements}

The authors would like to express their sincere thanks to the anonymous referees for their valuable comments and useful suggestions in improving the paper. The first author was supported partially by grant No. NSC 101-2115-M-017-001 of the National Science Council of the Republic of China. 


\section{References}

1. Du, W-S: A note on cone metric fixed point theory and its equivalence. Nonlinear Anal. 72(5), $2259-2261$ (2010)

2. Huang, LG, Zhang, X: Cone metric spaces and fixed point theorems of contractive mappings. J. Math. Anal. Appl. 332 1468-1476 (2007)

3. Taylor, AE, Lay, DC: Introduction to Functional Analysis, 2nd edn. Wiley, New York (1980)

4. Chen, GY, Huang, XX, Yang, XQ: Vector Optimization. Springer, Berlin (2005)

5. Du, W-S: On some nonlinear problems induced by an abstract maximal element principle. J. Math. Anal. Appl. 347(2), 391-399 (2008)

6. Abdeljawad, T, Karapınar, E: A gap in the paper 'A note on cone metric fixed point theory and its equivalence' [Nonlinear Anal. 72(5), (2010), 2259-2261]. Gazi Univ. J. Sci. 24(2), 233-234 (2011)

7. Bakhtin, IA: The contraction mapping principle in quasimetric spaces. Funct. Anal. 30, 26-37 (1989)

8. Czerwik, S: Nonlinear set-valued contraction mappings in b-metric spaces. Atti Semin. Mat. Fis. Univ. Modena 46, 263-276 (1998)

9. Akkouchi, M: A common fixed point theorem for expansive mappings under strict implicit conditions on $b$-metric spaces. Acta Univ. Palacki. Olomuc., Fac. Rerum Nat., Math. 50(1), 5-15 (2011)

10. Aydi, H, Bota, M-F, Karapınar, E, Moradi, S: A common fixed point for weak $\phi$-contractions on $b$-metric spaces. Fixed Point Theory 13(2), 337-346 (2012)

11. Hussian, N, Shah, MH: KKM mappings in cone b-metric spaces. Comput. Math. Appl. 62, 1677-1684 (2011)

12. Shi, $L, X u, S$ : Common fixed point theorems for two weakly compatible self-mappings in cone $b$-metric spaces. Fixed Point Theory Appl. 2013, 120 (2013)

13. Huang, $\mathrm{H}, \mathrm{Xu}$, S: Fixed point theorems of contractive mappings in cone $b$-metric spaces and applications. Fixed Point Theory Appl. 2013, 112 (2013)

14. Pacurar, M: A fixed point result for $\varphi$-contractions on $b$-metric spaces without the boundedness assumption. Fasc. Math. 43, 127-137 (2010)

doi:10.1186/1687-1812-2013-210

Cite this article as: Du and Karapınar: A note on cone $b$-metric and its related results: generalizations or

equivalence?. Fixed Point Theory and Applications 2013 2013:210.

\section{Submit your manuscript to a SpringerOpen ${ }^{\circ}$ journal and benefit from:}

- Convenient online submission

- Rigorous peer review

- Immediate publication on acceptance

- Open access: articles freely available online

- High visibility within the field

- Retaining the copyright to your article 